\title{
Development of the Backscattered Intensity Integral Imaging Technique for the Detection of Micro-Channels in Shelf-Stable Food Packages
}

\author{
Kay Raum and William D. O'Brien, Jr. \\ Bioacoustics Research Laboratory, Department of Electrical and Computer Engineering, University of Illinois, \\ 405 North Mathews Avenue, Urbana, IL 61801 (Current address for KR: Institute of Medical Physics and \\ Biophysics, Martin-Luther-University Halle-Wittenberg, D-06097 Halle/Saale, Germany)
}

Abstract - Hermetically sealed flexible food packages require a very effective seal integrity test to guarantee the quality of thermally processed food. In this contribution a new ultrasonic pulse-echo technique called Back-scattered Intensity Integral (BII)-mode imaging for the detection of microchannel defects in these types of food packages is proposed and evaluated. The BII technique integrates the received backscattered signal of each Aline and produces an integrated spatial map, a $B I I$ mode image. It has been shown that the $B I I$-mode image has the ability for subwavelength detection of channel defects. For example, that a $10 \mu \mathrm{m}$ diameter channel defect can be detected at a center frequency of $15 \mathrm{MHz}(\lambda \approx 140 \mu \mathrm{m})$.

\section{INTRODUCTION}

Retort pouches are a hermetically sealed flexible package in which food is placed and thermally processed to produce a shelf-stable product. The package remains sealed after processing, so the advantages of metal cans and plastic boil-in bags are combined [1-3]. Currently §CFR 381.301(d) requires that producers manually inspect each container, once after sealing and again after retorting. The cost, variability and slow speed of this inspection procedure have restricted the use of these types of packages to niche markets such as specialty diet foods and military rations.

Channel defects as small as $10 \mu \mathrm{m}$ can propagate some types of micro-organisms. Human observer inspection capabilities, at their best, are in the range of $50 \mu \mathrm{m}$ so there is a critical need to develop a reliable methodology to detect, and possibly characterize, channel defects smaller than 50 $\mu \mathrm{m}$. Various non-destructive techniques including machine vision imaging, infrared imaging, X-ray, MRI and spectro-photometer have been evaluated. Only X-ray and MRI can identify defects smaller than $50 \mu \mathrm{m}$, but both techniques fail under certain conditions, for instance if the defect is air-filled [4-6].

A recent study using a Scanning Laser Acoustic Microscope (SLAM) operating at 100 $\mathrm{MHz}$ showed that defects as small as $10 \mu \mathrm{m}$ can be detected [7]. In this study, simulated channel defects in the range from 9 to $325 \mu \mathrm{m}$ in diameter in transparent plastic film with a total thickness of about $220 \mu \mathrm{m}$ were inspected using a pulse-echo technique at frequencies of about 15 and $20 \mathrm{MHz}$. A new imaging technique called Backscattered Intensity Integral $(B I)$-mode imaging for the detection of defects was developed and evaluated.

\section{Sample Preparation and Validation}

Thirty-four samples with channel defects in a range from 9 to $325 \mu \mathrm{m}$ were prepared. In order to simulate a high and low contrast case, the channels were filled with either air or water. Plastic trilaminate microwavable retort pouch (Fuji Tokushu Shigyo Co. Ltd., Seto Aichi, Japan) was used as a packaging material. The propagation speed in the plastic laminate is $2380 \mathrm{~m} / \mathrm{s}$ [7]. This material is used commercially in Japan and was chosen for this study due to its transparency. Generally the retort pouch can contain an aluminum layer or the surface of the package might be printed. Each channel defect sample was prepared by placing a tungsten wire $(10,25,37,50,75,100$ or $220 \mu \mathrm{m}$; California Fine Wire Company, Grover City, CA) between two layers of the trilaminate material. An automatic heat sealer (Doboy HSC42051, Doboy Co., New Richmond, Wisconsin) was used to seal the tungsten wire into place at a sealing temperature of $132^{\circ} \mathrm{C}$. After five minutes the wire was pulled out. In order to fill the channel with water, the wire was removed, while the sample was submersed in a water bath. Finally both ends of the channel were sealed to keep either air 
or water inside. Small needle pricks were placed on opposite sides of the channel as reference marks for both the ultrasound images and the validation light transmission microscope images.

Light transmission microscope images with a calibration grid (Reichert Jung: $2 \mathrm{~mm}$ divisions into subdivisions of $10 \mu \mathrm{m}$ ) were used to determine the exact cross-sectional channel defect dimensions. Images were captured using a Nikon Optiphot-2 light microscope, Sony CCD color video camera, RasterOps frame-grabber board, Macintosh IIci computer, and Adobe Photoshop software. The image area was 420 by $316 \mu \mathrm{m}$ for the crosssectional images and 2.1 by $1.6 \mathrm{~mm}$ for the topview images, respectively.

\section{System Description}

The channel-defect sample was placed in a water tank $\left(\approx 20^{\circ} \mathrm{C}\right)$ with the defect oriented approximately normal to the sound beam direction. Fifteen and $20 \mathrm{MHz}$ spherical focused ultrasonic transducers with measured center frequencies of 13 and $17 \mathrm{MHz}$ (Panametrics V319 and V317, respectively, Waltham, MA) were excited by a computer-controlled pulser/receiver (Model 5800, Panametrics, Waltham, MA). The received echo signal was amplified (20 dB), band-pass filtered (1$35 \mathrm{MHz})$ and displayed $(500 \mathrm{Ms} / \mathrm{s})$ on a digitizing oscilloscope (Tektronix Model 11401) with a 10bit resolution.

The image data were produced by moving the transducer in a rectangular grid pattern parallel to the sample surface with a computer-controlled micro-precision positioning system (Daedal Inc., Harrison City, PA) which has a positional accuracy of $2 \mu \mathrm{m}$. The incident direction of the ultrasound beam was intentionally oriented at a known angle either normal or slightly oblique $\left(\leq 10^{\circ}\right)$ to the plastic surface (small wrinkles in the surface were neglected) yielding an angular separation between reflected and refracted waves. Positioning system, oscilloscope and pulser/receiver were connected to a GPIB-board and controlled by a $486-66$ PC. The grid spacings in the directions perpendicular and parallel to the axis of the channel defect were 30 $\mu \mathrm{m}$ and $100 \mu \mathrm{m}$, respectively, for a lateral 3 by 4 $\mathrm{mm}$ field-of-view. The number of A-scan locations per image was 4000 ( 100 by 40 ). This 3-D data set containing all 4000 512-point A-scans were stored to the hard disk and transferred to a SUN Sparc 20 workstation for off-line processing. All computations were performed in MATLAB (The MathWorks, Inc., Natick, MA).

\section{Backscattered Intensity Integral}

Theoretical resolution limits for timeinstantaneous acoustic images at 15 and $20 \mathrm{MHz}$ are not sufficient to resolve features in the dimension of interest (e.g., axial and lateral resolution $\approx$ $180 \mu \mathrm{m}$ at $20 \mathrm{MHz}$ [8]). Conventional B-mode images resolved the sample surfaces, but the layered structure of the laminate as well as the channel defects were not visible. However, a characteristic shadow at the location posterior to the expected channel was observed especially for the larger defects [9]. Also, B-mode technique itself has only conditional application for the intended purpose of on-line channel detection. In order to detect channel defects and to distinguish them from other discontinuities within the material, information about the defect's spatial extent is necessary. This need led to the proposed $B I I$-mode imaging technique.

The characteristic shadow region in the Bmode images suggests that any discontinuity in the plastic layer could affect the amplitude of the received RF echo signal and possibly the total reflected echo energy. This can be quantified by integrating each absolute value of the unfiltered Hilbert-transformed RF echo signal, that is,

$$
B I I=\int_{t_{1}}^{t_{2}}[e n v(R F(t))] d t
$$

where $B I I$ is called a Backscattered Intensity Integral. Since the Hilbert-transformed signal represents the envelop of the RF-signal amplitude, the $B I I$ value is proportional to the square root of the backscattered energy. The integration boundaries $t_{1}$ and $t_{2}$ define times prior to and beyond the locations of the sample top and bottom, respectively, that is, the times between when the envelop of the received signal is nonzero. Since the envelop of the signal is zero before and after the echoes from the sample occur, a wide time interval, for instance the entire focal zone can be chosen for the integration boundaries. Unlike a C-mode image for which the amplitude of a certain depth within the sample has to be used to process the image, the $B I I$ time inter- 
val includes the entire sample thickness. Thus, $B I I$ is relatively insensitive to changes in the probesample distance and no information about any variations in the sample structure, such as channel defects, inclusions, delaminations or even wrinkles in the sample surface is lost. In Fig. 1, where a 95 $\mu \mathrm{m}$ channel defect is shown, the needle marks and wrinkles combined with delaminations are also clearly visible.

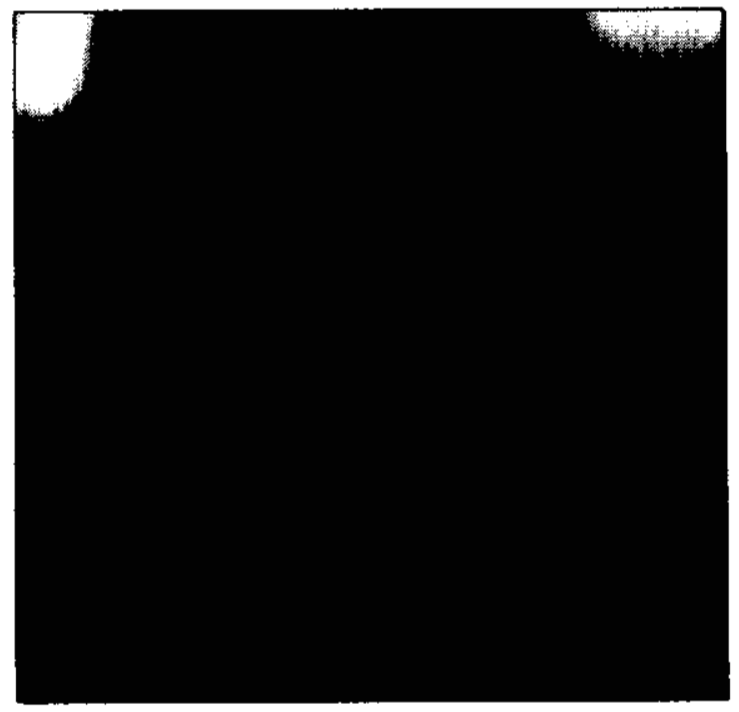

Figure 2: Normal-incident BII-mode image of a 95- $\mu \mathrm{m}$ water-filled channel defect. The reference needle marks are seen as dark-spot features on each side of the channel. The bright spots on both upper corners are delaminations where the two trilaminate plastic layers were not sealed.

The $B I I$-mode images were processed from the entire acquired 3-D data set. To generate a BIImode image, every A-scan was processed (Eq. 1) to yield unscaled $B I I$ values. The two-dimensional BII-data set was then interpolated by a factor of two in the direction perpendicular to the channel axis and by a factor of seven in the direction parallel to the channel axis to yield 15.0 by $14.3-\mu \mathrm{m}$ pixel sizes. Finally, the matrix was median filtered and displayed as a two-dimensional gray-scale map (Figs. 1 and 2a).

\section{RESULTS}

Air-filled and water-filled channel defects as small as $10 \mu \mathrm{m}$ could be detected in the $B I I$ mode images at $20 \mathrm{MHz}$ under oblique-incident conditions (Fig. 2b). At $15 \mathrm{MHz}, 10-\mu \mathrm{m}$ air-filled and $20-\mu \mathrm{m}$ water-filled channel defects appeared to be minimally detectable. It should be noted that the observed channel size is larger than the actual channel defect size. This is due to the beam width being larger than the channel defect diameter and therefore the spatial extent of the defect image is, in fact, the beam width.

The difference in the $B I I$-mode image between a region of the channel defect and its adjacent undisturbed region is quantified from

$$
\Delta B I I=B I I_{\text {undisturbed }}-B I I_{\text {extreme }}
$$

where $B I I_{\text {undisturbed }}$ is the $B I I$ value from the undisturbed region adjacent to the channel defect and $B I I_{\text {extreme }}$ is the extreme $B I I$ value from the approximate center location of the channel defect. The quantity $\triangle B I I$ can be thought of as a measure of contrast in the $B I I$-mode image. The dependence of $\triangle B I I$ as a function of the lateral channel diameter from normal-incident $B I /$-mode images obtained with a $20-\mathrm{MHz}$ probe was measured for both water-filled and air-filled channel defects, and a significant $\triangle B I I$ difference between whether the channel was air filled or water filled was not observed.

\section{SUMMARY}

A new $B I I$-mode imaging technique was developed and evaluated for detecting subwavelength $(\lambda \approx 140-180 \mu \mathrm{m})$ channel defects in plastic trilaminate microwavable retort pouch materials. $B I$-imaging technology at $20 \mathrm{MHz}$ appears to be capable to detect channel defects in the $10-\mu \mathrm{m}$ range regardless of whether low impedance (air) or high impedance (water) material resides within the channel defect. Combining this capability with a reasonable depth of penetration and the ability to inspect opaque materials, $B I I$-imaging technology has clear advantages compared to other nondestructive methods. At the ultrasonic frequencies evaluated, the capability of $B I I$-mode images already meets the requirements for safe food package inspection in terms of the critical channel size, that is, it can detect channel defects reliably for diameters less than $50 \mu \mathrm{m}$. Also, there is still the added potential to increase the ultrasonic frequency beyond that used in this study while, at the same 


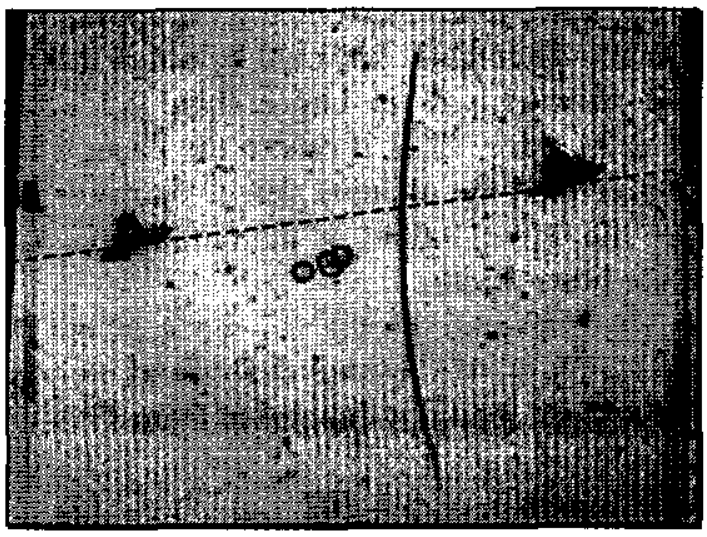

(a)

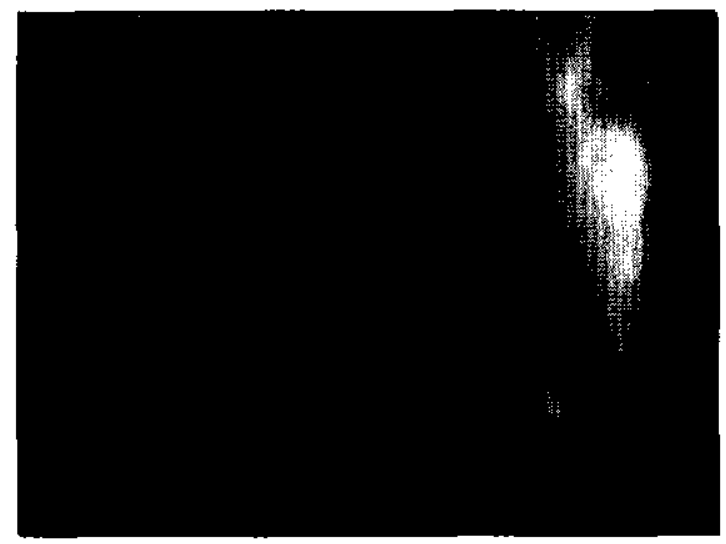

(b)

Figure 2: Light microscope image (a) and corresponding BII-mode image (b) of a 10- $\mu \mathrm{m}$ air-filled channel. In the light microscope image, the channel defect is the slightly arched vertical line, the dashed straight line indicates the location where the sample was cut for end-view light microscopy evaluation and the three roughly circular structures in the center are material imperfections. (b) $B I I$-mode image of the upper part of the sample after the cut, $20 \mathrm{MHz}$, oblique incidence.

time, maintaining a reasonable depth of penetration sufficient for common thicknesses of food packages.

\section{ACKNOWLEDGEMENTS}

This work was supported in part by the Value-Added Research Opportunities Program, Agricultural Experiment Station, University of Illinois. The authors would like to acknowledge the professional assistance with the sample validation by James F. Zachary, DVM, PhD, Department of Pathobiology, University of Illinois.

\section{REFERENCES}

[1] F. E. Long, "Flexible packages now withstand heat processing temperatures of foods," Package Engineering, vol. 7, no. 3, pp. 63-65, 69-72, 74, 76-77, 79-80, 1962.

[2] N. H. Mermelstein, "The retort pouch in the U.S.," Food Technology, vol 30, no. 2, pp 28-37, Feb. 1976.

[3] R. A. Lampi, G. L Schulz, T. Ciavarani and P. T. Burke, "Performance and integrity of retort pouch seals," Food Technology, vol. 30, no. 2, pp. 38-48, Feb. 1976.

[4] C. L.Harper, B. A. Blakistone, J. B. Litchfield and S. A. Morris, "Developments in food packaging integrity testing," Food Technology, vol.6, no. 10, pp.336-340, 1995.
[5] D. T. Maunder, J. F. Folinazzo and J. J. Killoran, "Bio-test method for determining integrity of flexible packages of shelf-stable foods," Food Technology, vol. 22, no. 5, pp. 615-618, May 1968.

[6] J. W. Szczeblowski and F. J. Rubinate, "Integrity of food packages," Modern Packaging, vol. 38, no. 10, pp. 131-134, 1965.

[7] A. A. Safvi, H. J. Meerbaum, S. A. Morris, C. L. Harper and W. D. O'Brien, Jr., "Acoustic imaging of defects in shelf-stable food packages," Journal of Food Protection, (in press).

[8] K. Raum and W. D. O'Brien, Jr., "Pulseecho field distribution measurement of highfrequency ultrasound sources," IEEE Transactions on Ultrasonics, Ferroelectrics and Frequency Control, (submitted).

[9] K. Raum, A. Ozguler, S. A. Morris and W. D. O'Brien, Jr., "Channel defect detection in shelf-stable food packages using highfrequency pulse-echo imaging" IEEE Transactions on Ultrasonics, Ferroelectrics and Frequency Control, (submitted) 